Moon, the Mohole project has not yet delivered a stick of upper mantle rock. Some of the best preserved mineral grains available to geologists at the present time from high pressure environments are in fact the crystalline inclusions in diamond; the commonest of these inclusions is olivine, and six olivines (three from Venezuela and three from Ghana) microprobed at the Geophysical Laboratory in Washington showed not only an extremely constant ratio $\mathrm{Fe} / \mathrm{Mg}$ (about 8 per cent $\mathrm{Fe}$ ), but also an extremely constant $\mathrm{Ni}$ content, at about 5 per cent of the Fe content. As the 1959 paper put it, "diamond can form in several different ways, and stubborn mysteries still surround some of them". Diamonds aside, geologists as a whole owe much to General Electric. Maybe it is time that somebody named a new mineral for them. - H. J. M.

\section{Sequencing DNA}

Methods, notably those devised and developed during the past several years by Sanger's group at the MRC Laboratory of Molecular Biology in Cambridge, which allow the determination of the nucleotide sequences of RNA molecules have become part of the stock in trade of molecular biology laboratories. Announcements of the determination of the complete base sequence of this or that transfer RNA or other small RNA molecule have thus become almost commonplace and cause only a ripple of general interest, although messenger RNA molecules, whose sequences molecular biologists would dearly like to know, cannot usually be purified to the standards required for sequence analysis. In short, the methodology for sequencing RNA molecules is at hand and the chief obstacles to analysing many interesting RNA molecules lie in their purification.

By contrast, methods for analysing the base sequences of DNA molecules are still in their early infancy and most groups intent on determining the nucleotide sequence of short fragments of DNA have shirked taking the bull by the horns and have instead adopted indirect approaches. Usually these methods involve either transcribing in vitro with Escherichia coli RNA polymerase the DNA of interest and then analysing the base sequence of the RNA transcript, or using $E$. coli DNA polymerase I to synthesize a labelled complementary DNA chain which can be analysed. The obvious disadvantage of these sorts of experiments is that they rely on the fidelity of in vitro transcription or DN'A synthesis, which is not the least of the reasons why Sanger and his colleagues have tackled the problem of devising a generally applicable methodology for the direct analysis of the base sequences of DNAs. To judge from what Ziff et al. and Robertson et al. had to say in last Wednesday's Nature New Biology (241, 34 and 38 ; 1973) they have succeeded in their objective.

The first problem facing anybody who tries to analyse the base sequence of a naturally occurring DNA molecule is that of devising methods for partially digesting the DNA into specific fragments small enough to analyse ; the smallest DNAs, phage genomes, are at least 5,000 bases long. Ziff et al. used T4 phage endonuclease, which preferentially attacks single-stranded DNA, to digest par- tially single-stranded $\phi \times 174$ DNA in conditions which favour the formation of DNA secondary structure. They then separated by gel electrophoresis the fragments of $\phi X 174$ DNA, which because of the specificity of T4 endonuclease have a $5^{\prime}$ cytidine residue bearing a $5^{\prime}$ phosphate group and isolated two for further analysis. Robertson et al., on the other hand, used a technique devised by Steitz for the sequence analysis of the ribosome binding sites of phage R17 RNA to obtain a fragment of $\phi \times 174$ DNA. They bound $70 \mathrm{~S}$ ribosomes to the single-stranded $\phi X 174$ DNA, using conditions Bretscher had devised to bind ribosomes to DNA, and then they digested away all the $\phi \times 174$ DNA that was not protected by the bound ribosomes. The ribosomes were then dissociated and the protected fragments of DNA released were then subjected to gel electrophoresis and chromatography. The population of fragments of DNA proved to comprise one large portion about fifty nucleotides long and several smaller portions; the principal portion was isolated for further analysis. Clearly both these methods for obtaining small fragments of DNA have their limitations and no doubt in the future enzymes such as the bacterial restriction endonucleases, which can cut double-stranded DNA at specific sites, will be used by DNA sequencers.

Having obtained a fragment of $\phi X 174$ DNA of manageable size, Ziff et al. began the task of analysing its sequence. The procedure which they devised involves depurination and further digestion of the fragment with T4 endonuclease, snake venom phosphodiesterase and spleen phosphodiesterase coupled with standard firgerprinting chromatography. Using these procedures the group obtained the base sequences of a series of overlapping oligonucleotides and hence the sequence of the forty-eight nucleotides in the fragment of $\phi \times 174$ DNA.

By exploiting these procedures and in addition using streptococcal nuclease Robertson et al. determined the sequence of the fifty-one bases of the strong ribosome binding site in $\Phi$ X174 DNA which they had isolated. This sequence is interesting because it contains an ATG triplet which is, of course, the equivalent of an AUG codon in messenger RNA. Furthermore, if the base sequence is read in triplets, starting with the ATG triplet, an amino-acid sequence can be predicted. This predicted sequence is identical to that at the amino-terminal end of the $\phi \times 174$ spike protein specified by cistron $G$ which Air and Bridgen determined once Robertson et al. had begun the analysis of their fragment of $\phi$ X174 DNA. Together these results unambiguously prove that the fragment of the $\phi \times 174$ genome which Robertson et al. have sequenced is the beginning of the $G$ cistron.

This sequence, as Robertson et al. point out, has several striking features in common with the sequences of some of the ribosome binding sites of the RNA phages R 17 and $\mathrm{Q} \beta$. As they say, "Thus a pattern of similarities seems to be emerging among known ribosome binding site sequences which may in the future be explained in terms of ribosome or factor specificity and thus play an important part in the control of initiation of protein synthesis". But if realization of the significance of these initiation site sequences is something for the future, the methods that Ziff and Robertson and their colleagues have pioneered can be immediately exploited to yield information about the base sequences of many DNAs, for they have the twin advantages of being direct and being generally applicable. -From a Correspondent. 